\title{
试验仪表系统在 DCS 实现可行性分析
}

\section{Analyze on feasibility of DCS intergradation KME system}

\author{
王绪霄 赵友有 陈源杉, \\ XuXiao Wang Youyou Zhao Yuanshan Chen \\ 中广核研究院有限公司 中国·广东深圳 518031
}

China Nuclear Power Technology Research Institute (CNPRI), ShenZhen, Guangdong, 518031, Chin

\begin{abstract}
摘 要: 压水堆核电站部分系统因特殊功能和性能要求采用了独立控制系统，包括试验仪表( KME) 系统。随着数字化 技术发展，核电站 DCS 系统能够提供各类控制、保护手段及监测信息，满足更多的应用场景。为简化核电站控制系统设置， 提高全厂信息化，本文主要研究试验仪表系统在 DCS 实现的可行性。

Abstract: PWR nuclear power plants adopt independent control system due to special function and performance requirements, including KME system. With the development of digital technology, DCS can provide various kinds of control, protection and monitoring functions to meet more application scenarios. In order to simplify the nuclear power plant control system and improve the whole plant informatization, this paper studies the feasibility of DCS intergradation KME system.
\end{abstract}

关键词: 核电站; DCS；试验仪表

Key words: Nuclear power station; DCS; KME

DOI: 10.36012 /etr.v2i9.2675

\section{1 引言}

由于压水堆核电站控制系统的经过了从模拟控制系统 到数字化控制系统的发展, 部分专用功能采用了独立的控制 系统, 包括但不限于 $\mathrm{KME}$ 系统。 $\mathrm{KME}$ 系统主要功能是在 核电站整个运行期间进行自动或定期的试验, 以提供设备性 能的信息或某些特定参数的任何可能的偏离。目前核电站均 设有试验仪表独立系统, 随着数字化控制技术的发展, 快速 数据转换、高精度采集、数据记录分析、日志报表、趋势等 方面功能更加完善。核电站 DCS (数字化仪控系统) 平台 能够提供各类控制、保护手段及监测信息，保证电站安全、 可靠和经济地运行。将 KME 系统功能在 DCS 中实现, 有 利于电厂运行、维修、管理等各部门对工艺数据的有效利用, 简化核电站控制系统设置。

\section{DCS 基本配置}

根据仪控功能的安全分级划分, 核电站 DCS 系统包括 安全级 DCS 以及非安全级 DCS : 安全级 DCS 主要执行反
应堆保护系统功能，用于应对设计基准事故，当保护参数达 到或超过设定值时, 触发紧急停堆、启动专设安全设施及其 他事故后缓解措施，保护三大核安全屏障 (燃料包壳、一回 路压力边界和安全壳）的完整性，使实验堆达到安全停堆状 态 ; 非安全级 DCS 实现全厂自动化控制, 在正常运行工况 下提供监视和自动控制功能，为操纵员提供足控的控制方 式、信息和操作指导, 是核电站仪控系统主要组成部分。

KME 系统安全等级为非安全级, 不要求抗震可用性, 以下考虑将 $\mathrm{KME}$ 系统在非安全级 DCS 平台实现。非安全 级 DCS 系统按照功能划分主要包括四个部分，采集与控制 系统、数据处理与服务系统、操作员系统和维护诊断系统。 不同 DCS 产品供应商有不同平台架构, 以广利核 SH-N 产 品为例, 非安全级 DCS 主要组成如下:

采集与控制系统属于 LEVEL1 层，可以分为控制站、 通讯站、LEVEL1 网关、网络和配电等设备。数据处理与服 务系统属于 DCS LEVEL2 层, 主要包括实时服务器、历史 服务器、LEVEL2 网关和相关网络系统。操作员系统属于

【作者简介】王绪霄（1990 ），女，河北衡水人，工程师，从事核电站仪控设计与技术改进研究。 
DCS LEVEL2 层, 主要包括操作员站、大屏幕显示系统和 相关网络系统。维护系统主要用于系统组态修改、系统配置 管理和系统故障诊断。

\section{$3 \mathrm{KME}$ 主要功能}

KME 试验仪表系统主要进行热平衡计算试验, 根据核 电站一二回路的热平衡得到反应堆一回路热功率, 由于仪表 本身的精度很高, KME 热功率计算是核电站反应堆功率计 算最准确的方法。使用 $\mathrm{KME}$ 计算得到的热功率, 利用功率 与流量的计算关系得到环路流量和通过反应堆压力容器的 流量。此外, KME 功能还包括利用换热器出入口温度以及 流量计算出热交换器性能参数, 判断其换热性能。

KME 系统结构包括现场控制层、系统服务层和监测控 制层 3 个功能层次。现场控制层包括采集机柜、编组机柜及 相关配电设备, 系统服务层包括数据处理计算机及相关网络 设备, 监测控制层主要指操作台及网关计算机。

\section{4 系统集成分析}

随着数字化技术的发展, 核电站目前采用的 DCS 系统 在快速数据转换、高精度采集、数据记录分析、趋势、报表 等方面功能更加完整, 能够满足不同种类的工业自动化性能 指标要求。通过对核电站各工艺环节设备进行集中监视、控 制和管理, 使得全厂信息化成为可能, 有利于电厂运维管理 部门对工艺数据的有效利用, 提高运行安全可靠性以及管理 效率。核电站目前的 KME 功能采用专用仪控系统实现, 实 质上也是一套功能单一、系统规模小的 DCS 系统, 但是需 要独立配置的采集控制机柜、交换机服务器等设备却并没有 减少。将 KME 功能集成到 DCS 实现, 为某些紧凑型应用 提供了良好的可选方案。

KME 功能在 DCS 系统实现的考虑因素中, 由于系统架 构的差异性、系统气余方案、软件配置、通讯总线设计等均 可以灵活选型, 所以重点放在分析具体功能板件的性能指标 是否满足 $\mathrm{KME}$ 专用仪控的数据采集、处理和计算精度的要 求, 例如采样频率、信号精度、存储容量、系统规模、算法 实现、控制策略等方面。

\section{1 系统规模}

KME 系统的输入测点包括主给水压力、温度、流量, 主蒸汽压力、温度, 重要厂用水/设冷水换热器温度、压力、 流量信号等, 总数小于 50 个, DCS 系统测点数量可以上万, 测点数量远远小于 DCS 测点的规模。

\section{2 数据采集处理}

KME 系统用于热平衡计算和热交换器计算的所有测量 信号为 :

1 ) 流量、压力测量变送器, 4 20Ma DC 信号, 两线制;

2 ) 温度传感器和变送器信号, 4 线制 Pt100 铂电阻温 度传感器, 变送器输出 4 20mADC 信号, 两线制;

3 ) RPN 核测量信号, 0 10V。

KME 系统精度应优于 $1 \%$, 对采集过程精度要求优于 $0.02 \%$, 最小采集周期 $25 \mathrm{~ms}$ 。目前 DCS 对系统精度无要求, $4 \sim 20 \mathrm{~mA}$ 信号采集精度 $0.1 \%$, 最小采集周期 $20 \mathrm{~ms}$ 。DCS 性 能不满足试验仪表的高精度采集要求，考虑开发高精度模拟 量采集板卡可以实现 KME 需求。且 DCS 在电气厂房的运 行环境中, 整个采集和输出通道的精度漂移保证 24 个月内 不需要对系统精度进行手动校准。

\section{3 算法实现}

纳入 DCS 的工艺系统控制过程多为标准化控制逻辑, 控制站的主控制器中由模块化的程序完成。KME 数据的处 理计算要求复杂，为了计算的关键指标得到保证，不适宜在 主控制器中部署; DCS 的计算服务器采用四核处理器, 功 能包括全局计算, 能够实现电站状态、性能计算、安全参数 计算等, 也可以满足 KME 计算过程的专用定制要求。

\section{4 时钟同步}

KME 系统为了保证关键指标能够为机组的经济运行提供 参考, 对数据采集的同步性有一定要求, 应能够接收电厂的时 钟脉冲, 对其内部时钟同步。DCS 系统已经有成熟的校时准则, 通过在管理网连接电厂外部校时源, 校时服务器校准系统内其 余服务器、操作员站、网关、控制站等设备。为避免数据传输 延迟影响时间分辨率, 在尽可能靠近信号源出将时标附加到 信号上，对于 AI 信号将在采集的 CPU 中打时间标签。

\section{5 软件功能}

KME 系统对测量参数选择、局部扫描, 数据记录在硬 盘上, 参数或实验结果显示与打印等功能均可以通过 DCS 系统的操作员系统、维护系统完成。KME 系统还通过网关 与 KNS 系统进行通讯，将实时数据和历史数据传送给电厂 实时信息监控系统；DCS 系统与 KNS 已经有通讯接口，实 时数据每秒刷新, 历史数据每 10 分钟下载一次, 通讯周期 能够满足 KNS 要求, 将相关数据配置在通讯点表中即可。

由上可见, $\mathrm{KME}$ 系统的主要特点是模拟量采集精度要 (下转第 80 页) 